\title{
Lessons Learned \\ in the \\ First Year Operating \\ Software Defined Radios in Space
}

David Chelmins, Dale Mortensen, Mary Jo Shalkhauser, Sandra K. Johnson, and Richard Reinhart

dchelmins@nasa.gov

NASA Glenn Research Center (GRC) 


\section{Overview}

- SCaN Testbed and Software Defined Radio

- SCaN Testbed SDRs

- SCaN Testbed Communications Paths

- Lessons Learned

- Characterize the Platform

- Mitigate Old Hardware

- Flexible Commands

- Flexible Telemetry

- Great Engineering Models

- Help Third-Party Developers

- STRS \& SCaN Testbed Solicitations 


\section{SCaN Testbed}

- Space Communications and Navigation (SCaN) Testbed

- Software-defined radio (SDR) research testbed

- Launched July 2012 to the International Space Station (ISS)

- Space Telecommunications Radio System (STRS) architecture

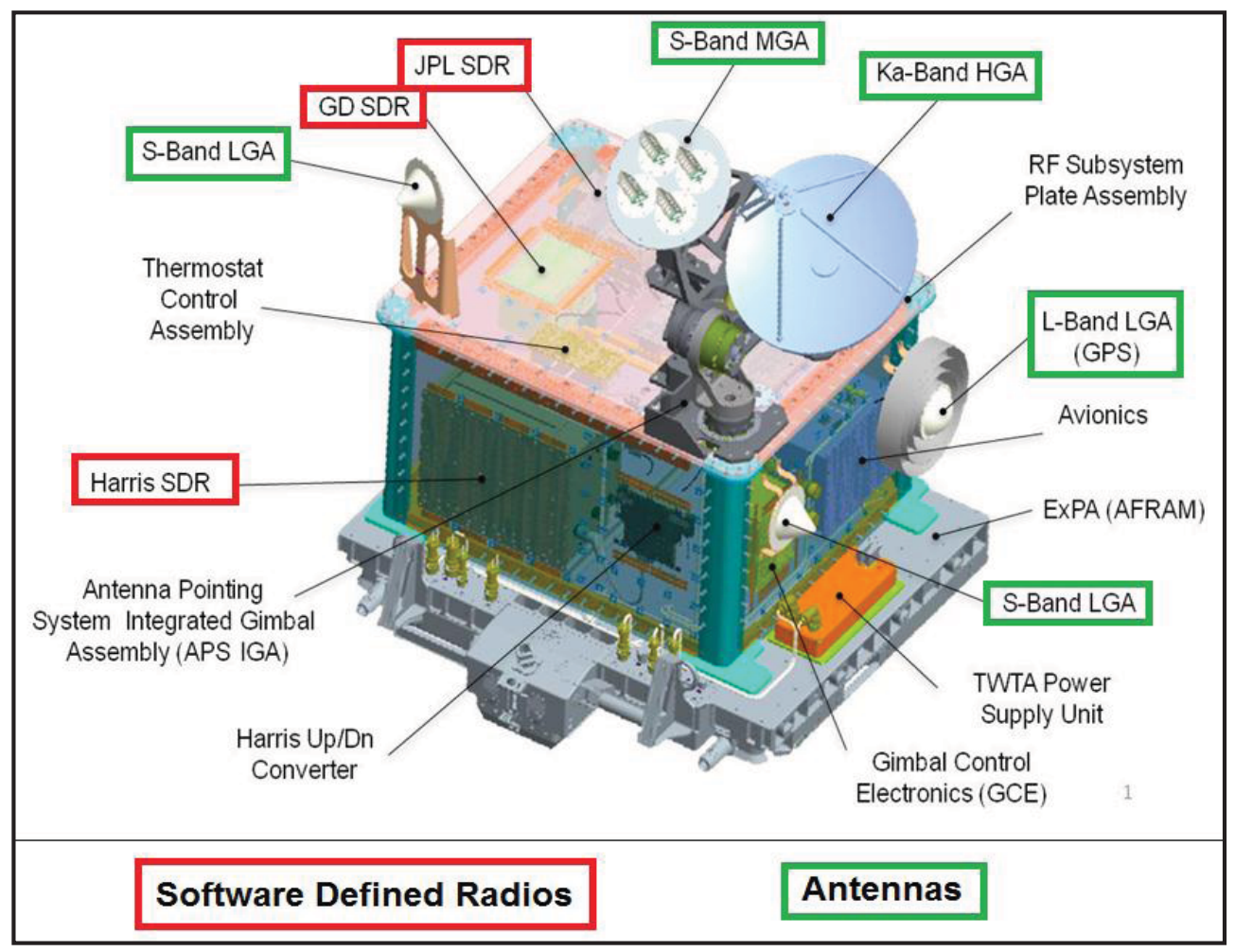

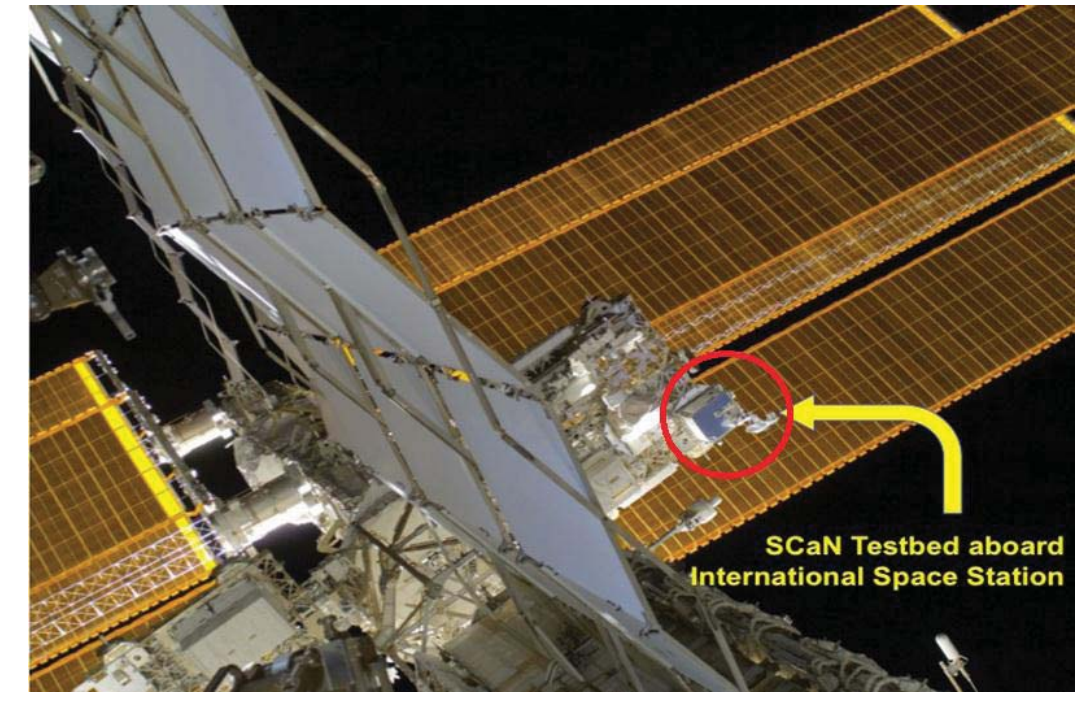

Above: SCaN Testbed

Left: SDRs and subsystems 


\section{SCaN Testbed SDRs}

- General Dynamics (GD) SDR

- 60 MIPS Coldfire (VxWorks) and (1) QPRO FPGA

- S-Band transceiver $(2.0-2.3 \mathrm{GHz})$ with $8 \mathrm{~W}$ amp

- $1 \mathrm{M}$ chalcogenide non-volatile phase-change memory

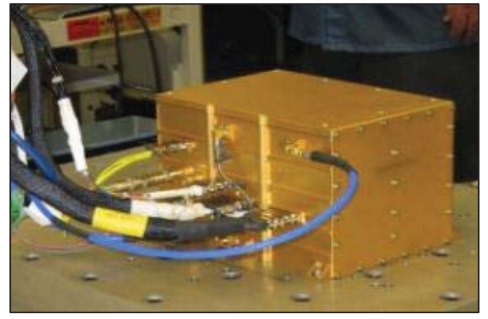

- Jet Propulsion Laboratory (JPL) / L3-CE SDR

- $66 \mathrm{MHz}$ SPARC (RTEMS) and (2) Virtex2 FPGAs

- S-Band transceiver $(2.0-2.3 \mathrm{GHz})$ with $7 \mathrm{~W}$ amp

- L-Band receiver at L1, L2, and L5 GPS frequencies

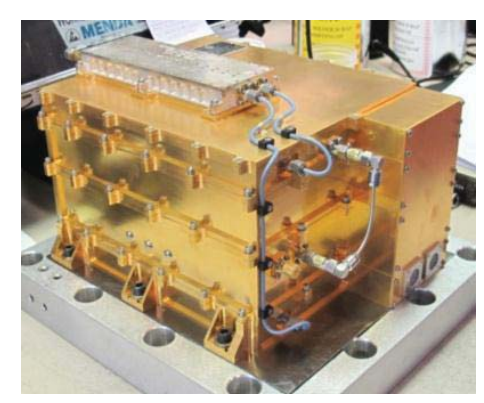

- Harris Corporation SDR

- 700 MIPS PowerPC (VxWorks) and (4) Virtex4 FPGAs

- Ka-Band transceiver $(22-26 \mathrm{GHz})$ with 40W TWTA

- Texas Instruments digital signal processor (DSP)

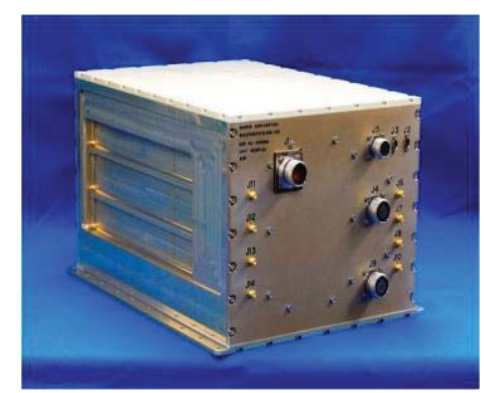




\section{SCaN Testbed Communications Paths}

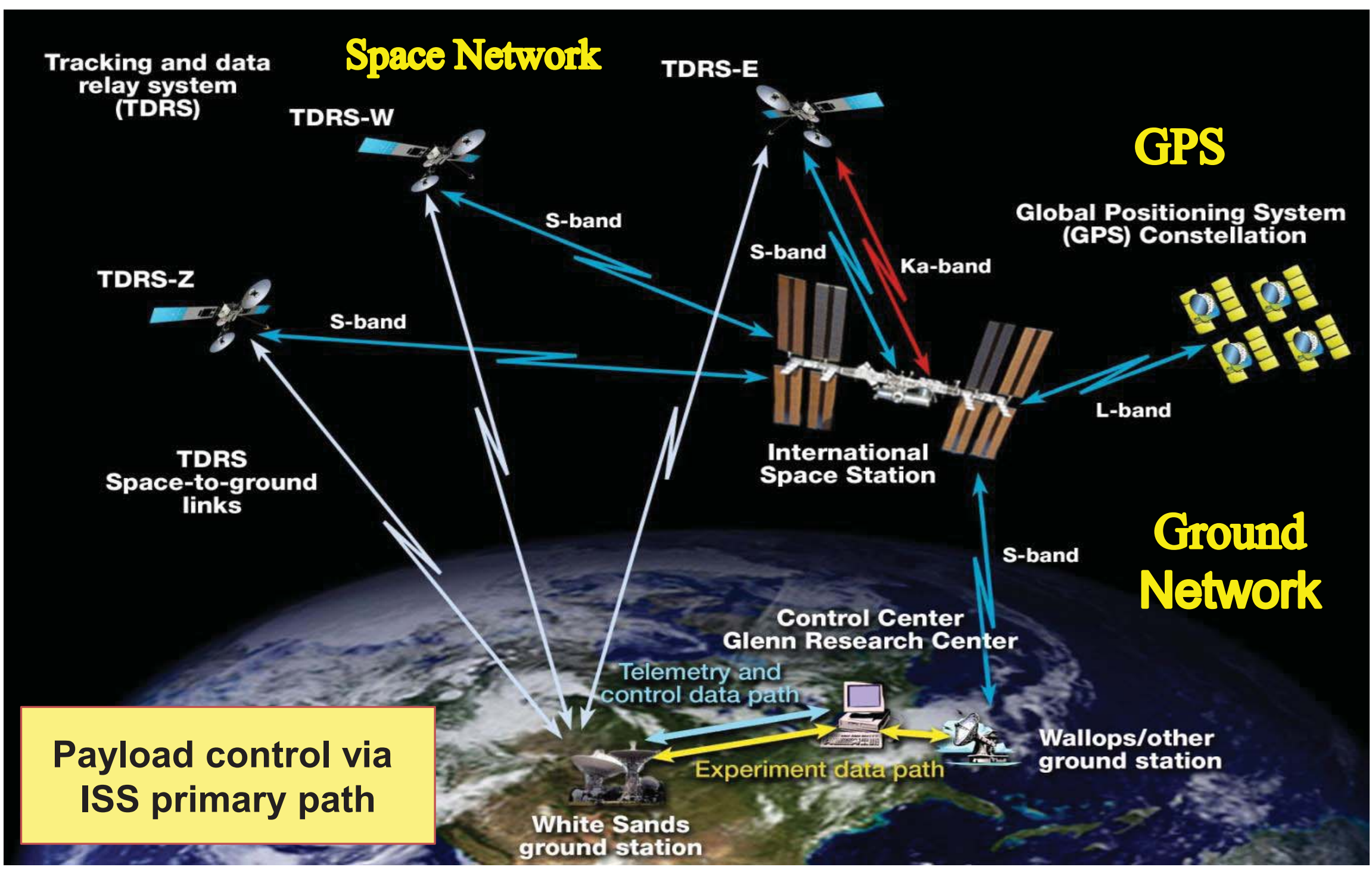




\section{LESSONS LEARNED}




\section{Characterize the Platform}

- Test the SDR hardware independent of the waveform (software)

- Development of a new waveform requires knowing platform performance.

- Low-level test waveforms are necessary for platform characterization.

- Store samples from the analog-to-digital converter

- Transmit samples out the digital-to-analog converter

- The operational waveform often is not the best tool for platform characterization

- Small subset of potential frequencies, modulations, and data rates

- Performance depends on waveform implementation

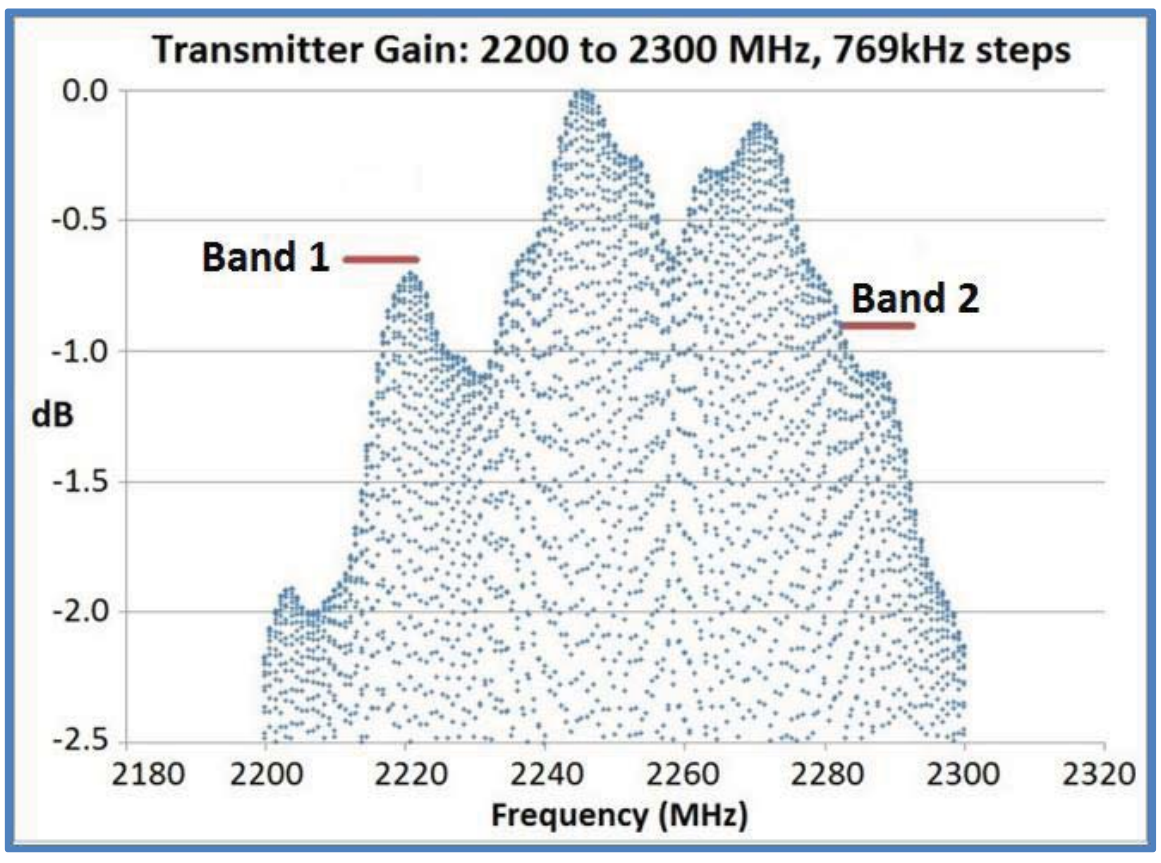

Require delivery of test waveforms to aid platform characterization 


\section{Mitigate Old Hardware}

- SCaN Testbed has several Xilinx Virtex 2 FPGAs

- Virtex 2 was last supported by Xilinx ISE 10.1 ( 2008)

- Increasingly challenging for present-day developers

- Old software libraries; vendors are less willing to fix bugs in old software

- Development boards are difficult to locate and buy

- Two perspectives

- Fly newer hardware - added risk due to unproven technology, but lower size/weight/power and enhanced functionality with slower obsolescence.

- Stay the course - use proven, low-cost, low-risk technology and find ways to accommodate future development without limiting mission duration.

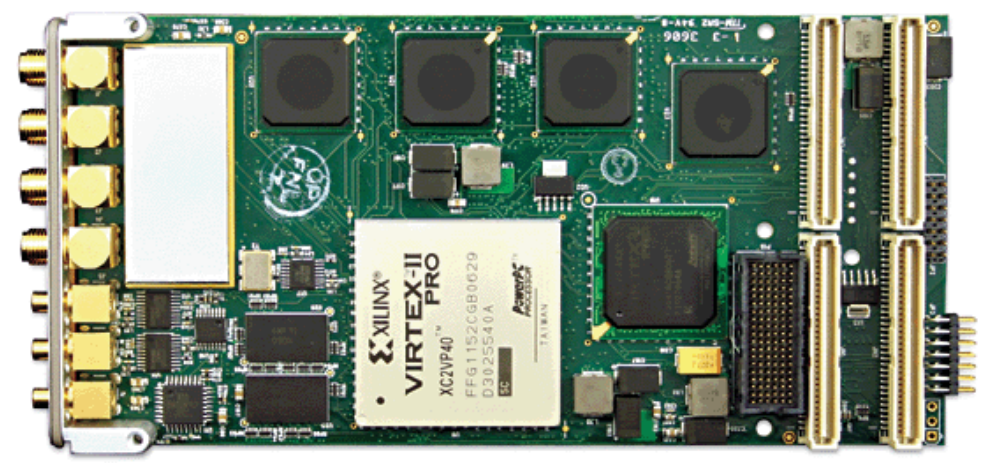

Fly both new and proven hardware to mix functionality with reliability 


\section{Flexible Commands}

- SDRs provide more command flexibility than traditional radios.

- How to effectively control and command SDRs?

- Commands - single operation, multiple operation, or scripts

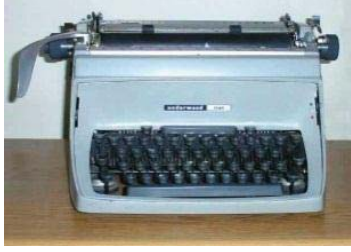

"Press a key"
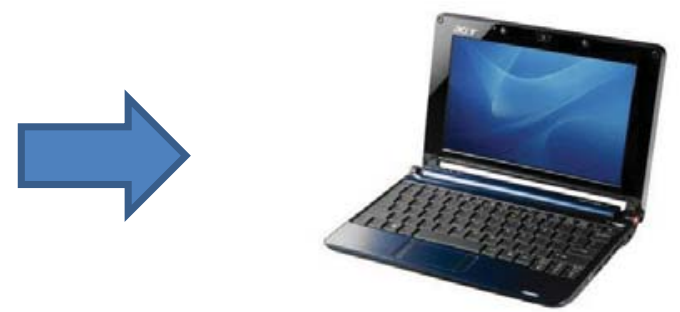

"Execute an operation"

- Flexibility requires an operations team with some radio knowledge

- Pre-defined command lists will grow over time, but fewer typos

- Effects of a "wrong command" can be larger with SDRs

Minimize the amount of "Human-in-the-Loop" to reduce mistakes.

Cost of flexibility is increased knowledge or training. 


\section{Flexible Telemetry}

- Telemetry can change with each waveform update

- Pre-defined fields are rigid - use name/value pairs or generic strings.

- Options to vary telemetry size, rate, contents, etc. on demand.

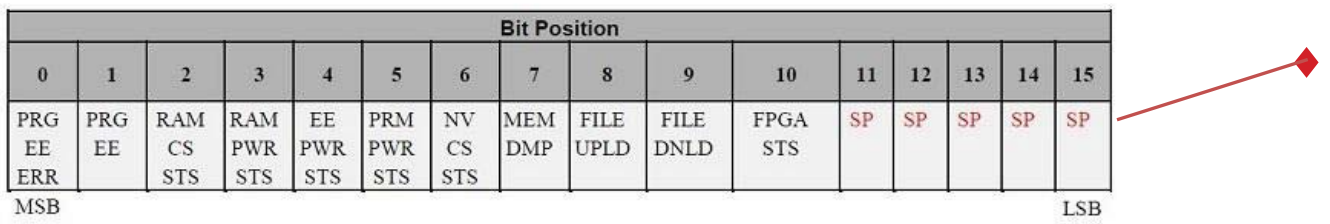

$<$ ?xml version $=" 1.0 "$ ? $>$

$<$ HarrisWaveFormPropertySettings >

<Property name="STRS_APP_STATE" value="" />

$<$ Property name $=$ "APQM_DB" value $=$ " " />

$<$ Property name $=$ "RX_CENTER_FREQ" value $="$ " $/>$

$<$ Property name $=$ "RX ATTEN VAL" value $="$ " />

$<$ Property name $="$ USE XML DEFAULTS" value $="$ " $/>$

$<$ Property name="WF_GPP_INFO" value $="$ "' / >

$<$ /HarrisWaveFormPropertySettings $>$

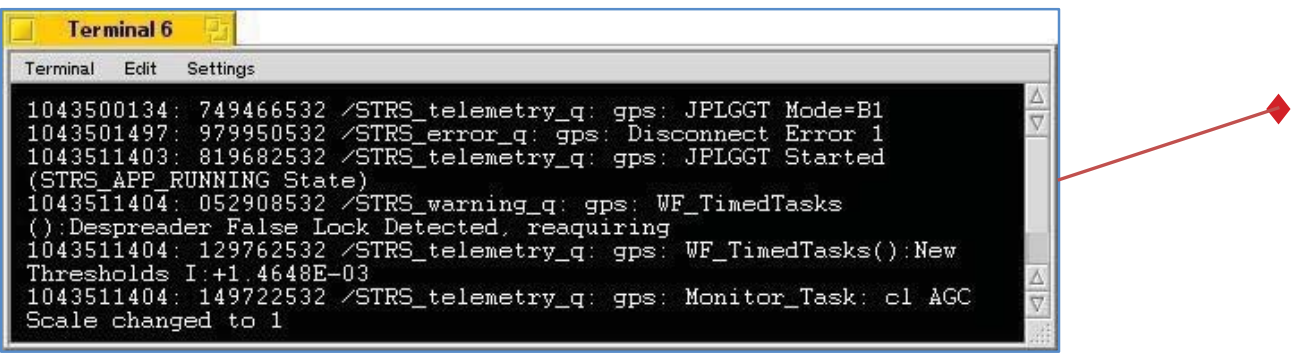

GD SDR 1553 data

- Fixed size, rate, message

- "Bit positions" and "Words"

Harris SDR name-value pairs

- Fixed rate, variable size/message

- Defined by XML.

\section{JPL SDR 1553 "serial" data}

- Variable size, rate, message

- Text-over-1553 telemetry 


\section{Great Engineering Models}

- High fidelity SDR engineering models (EMs) -> future waveforms

- Cost tradeoff: space-rated components vs commercial components

- Fidelity tradeoff: amplifiers/up-converters vs low-power baseband

- Performance tradeoff: antennas vs terminated test ports

- Case study - New Ka-band waveform

- Successful verification of command sequences on the ground

- Waveform worked half of the time on-orbit; otherwise, it crashed

- Traced issue to radio signal timing at temperatures below 14C

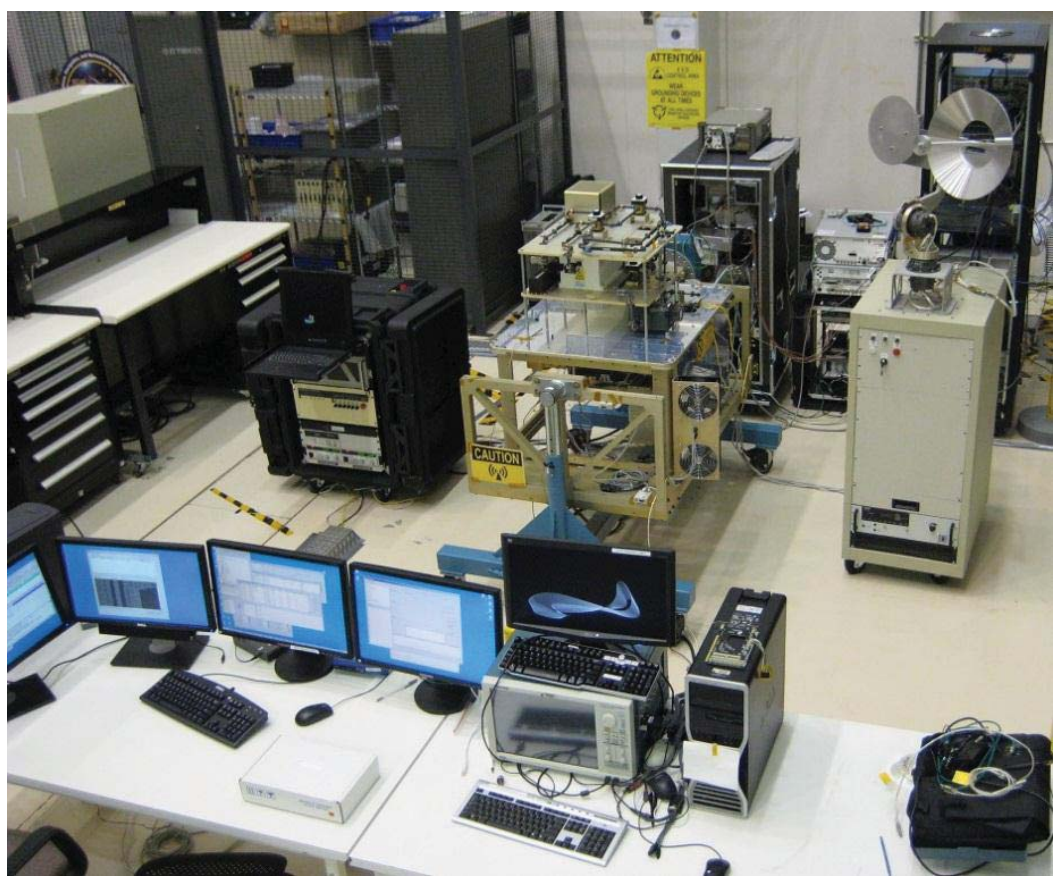

Invest in quality engineering models, but know differences/limitations 


\section{Help Third Party Developers}

- Waveform software should not depend on a specific platform

- STRS platforms come with an abstraction layer

- Why should a platform provider support a developer?

- Show that radio documentation is sufficient for $3^{\text {rd }}$ party software

- Platform developers are still involved as a service provider

- Proprietary documentation/code requires non-disclosure agreement

- Offer service/support agreements for $3^{\text {rd }}$ party development

- Is it possible for third party developers to write effective waveform code? Can they ever match/exceed what the platform manufacturer could have delivered? 
OPPORTUNITIES 


\section{STRS \& SCaN Testbed Solicitations}

- SDR Technology Request for Information

- Investigate the state-of-the-art of near-term and long-term, spaceapplicable SDR technology and concepts

- Understand the barriers to establishing a developer community to create or reuse applications for NASA communication systems

- Recommended updates to the STRS architecture: NASA-STD-4009.

- http://www.fbo.gov/ (NNC14ZRH014L, or search "STRS")

- SCaN Testbed Experiment Opportunities

- Focus on cognitive concepts for system efficiency (data throughput, power, and spectrum)

- Funded call for university experiments

- http://nspires.nasaprs.com/ (NNC12ZRH002C, or search "SCaN Testbed")

- Unfunded call for Space Act Agreements

- http://www.fbo.gov (Search "SCaNTestbed2014" posted in the last 365 days) 

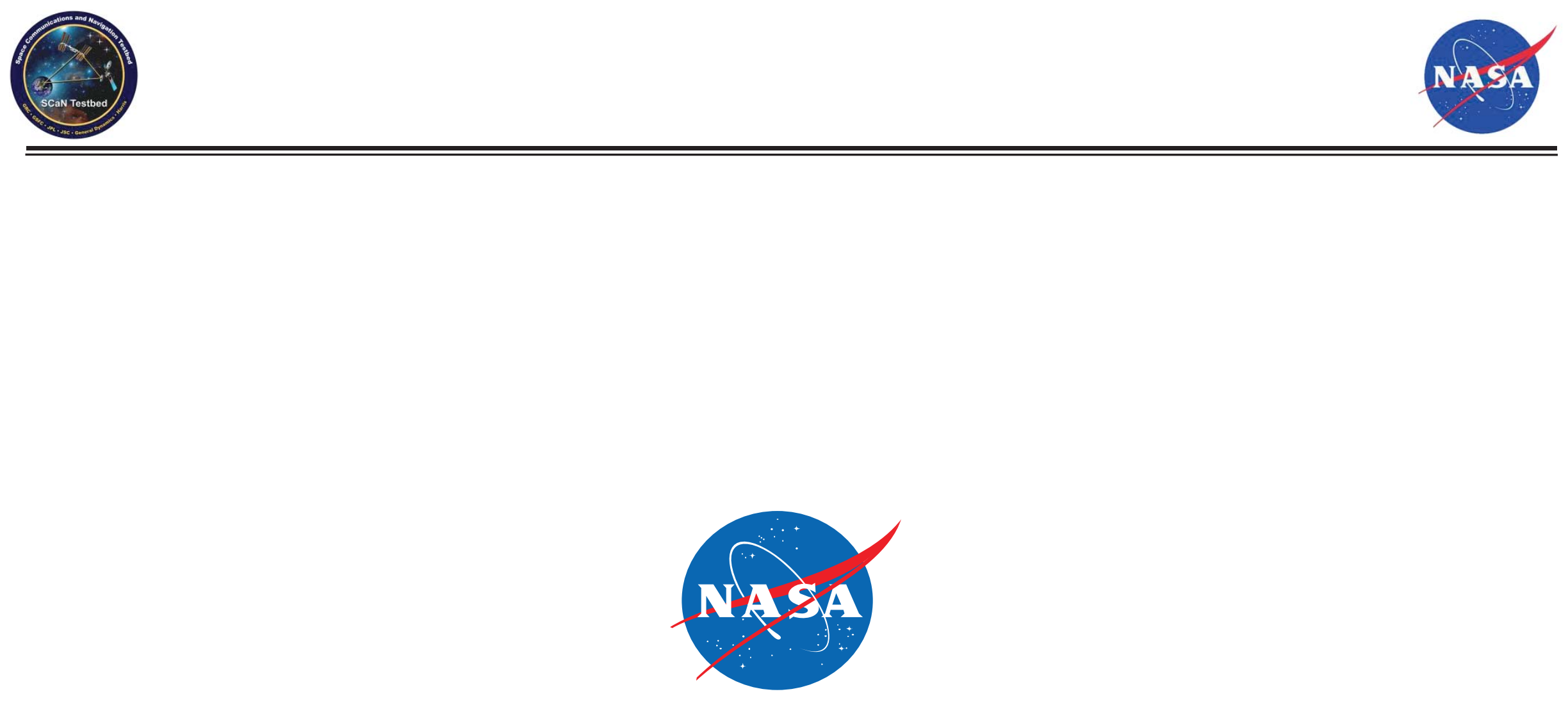

15 